\title{
Adenovirus E4 Protein
}

National Cancer Institute

\section{Source}

National Cancer Institute. Adenovirus E4 Protein. NCI Thesaurus. Code C129397.

A group of proteins encoded by the adenovirus early transcription locus 4 (E4). These

proteins are required for viral replication. 